\title{
Stability and limit cycles of a nonlinear damper acting on a linearly unstable thermoacoustic mode
}

\section{Journal Article}

Author(s):

Bourquard, Claire; Noiray, Nicolas

Publication date:

2019-05

Permanent link:

https://doi.org/10.3929/ethz-b-000345080

Rights / license:

In Copyright - Non-Commercial Use Permitted

Originally published in:

Journal of Engineering for Gas Turbines and Power 141(5), https://doi.org/10.1115/1.4042080 


\title{
Stability and Limit Cycles of a Nonlinear Damper Acting on a Linearly Unstable Thermoacoustic Mode
}

\author{
C. Bourquard ${ }^{\mathrm{a}}$, N. Noiray ${ }^{*, a}$ \\ ${ }^{a}$ CAPS Laboratory, Department of Mechanical and Process Engineering, ETH Zurich, Zurich 8092, Switzerland
}

\begin{abstract}
The resonant coupling between flames and acoustics is a growing issue for gas turbine manufacturers. They can be reduced by adding acoustic dampers on the combustion chamber walls. Nonetheless, if the engine is operated out of the stable window, the damper may be exposed to high-amplitude acoustic levels, which may trigger unwanted nonlinear effects. This work aims at providing an overview of the dynamics associated with those limit cycles using a simple analytical model, where a perfectly tuned damper is coupled to the combustion chamber. The damper, crossed by a purge flow in order to prevent hot gas ingestion, is modeled as a non-linearly damped harmonic oscillator, with vortex shedding as the main dissipation mechanism. The combustion chamber featuring a linearly unstable thermoacoustic mode is modelled as a Van der Pol oscillator. The fixed points of the coupled system and their stability can be determined by analyzing the averaged amplitude equations. This allows the computation of the limit cycle amplitudes as function of the growth rate of the unstable mode and the mean velocity through the damper neck. Simulink simulations are also performed and compared to the analytical predictions. Finally, experiments are performed on a simple rectangular cavity, where the thermoacoustic instability resulting from the interaction between heat release and acoustic pressure is mimicked by an electro-acoustic instability. A feedback loop is built, where the signal from a microphone is filtered, delayed, and amplified before being sent to a loudspeaker placed inside the rectangular cavity. The delay and gain of the feedback loop can be modified to change the growth rate of the instability. One Helmholtz damper can be added to the cavity and tuned to the unstable mode of interest. The stabilization capabilities of the damper and the amplitude of the limit cycle in the unstable cases are in good agreement between the experiments and the analytical and numerical predictions, underlining the potentially dangerous behavior of the system which should be taken into account for real engine cases.
\end{abstract}

\section{Introduction}

Over the last decades, the ever more stringent regulations on pollutant emissions have forced gas turbines manufacturers to design turbomachinery capable of running under leaner and leaner conditions. However, this lean combustion mode makes the combustor more prone to thermoacoustic instabilities, occuring when the fluctuating heat release from the flame interacts in a constructive

\footnotetext{
* Corresponding author

Email address: noirayn@ethz.ch (N. Noiray)
}

This is a pre-print version. Published in Journal of Engineering for Gas Turbines and Power (2019) Vol. 141(5), p. 051012 , DOI: $10.1115 / 1.4042080$ 
way with the acoustic pressure field. The limit cycles of such thermoacoustic instabilities have been the topic of multiple studies $[1,2,3,4]$. The suppression of thermoacoustic instabilities can be achieved by adding passive damping devices such as Helmholtz resonators to the combustion chamber $[5 ?, 6,7]$, but in practical system the determination of the ideal damper location can be quite challenging [8]. Using a linear model, the evolution of the stability limits $[9,10,11]$ and limit cycle amplitudes [12] of the system equipped with dampers has been studied. This model, using a linear dissipation term depending on the mean velocity of the purge flow going through the damper, is justified since dissipation occurs mostly because of vortex shedding at the damper exit $[13,14]$. However, if the acoustic pressure in front of the damper exceeds a threshold, the flow may reverse during part of the acoustic cycle, which then requires nonlinear modeling of the damping term. The nonlinear behavior of a Helmholtz damper has been studied on multiple occasions in the frame of damper impedance modeling, either with resistance depending only on amplitude $[15,16 ?]$, or adding a dependence on frequency in the model $[14,17,18,19]$. A study by Park and Sohn [20] investigated the damping of a cavity mode with a half-wave resonator at high amplitudes using numerical simulations. To the authors' knowledge, however, the influence of the Helmholtz resonator damping term nonlinearity on the stability of an acoustic mode has not been yet thoroughly investigated and is the topic of the present study. This is of utmost importance since the nonlinear damping term might lead to non-monotonic behavior such as sudden jumps to high-amplitude limit cycles, which can be dangerous for the machine integrity during ramping between operating points. In practice, reverse flow might lead to hot gas ingestion $[21,22]$ and thus detuning of the damper. The damper behavior can also be influenced by some other temperature or density difference between the damper and the cavity $[23,24]$. In the present study, however, only the influence of the damping nonlinearity is studied, and the damper is assumed to stay perfectly tuned at all conditions.

In a first part the analytical model is derived: the thermoacoustic instability is modelled as a Van der Pol oscillator, while the abovementionned nonlinear damping model is used for the resonator. Coupled differential equations are derived and averaged to obtain the theoretical limit cycle amplitudes for the perfectly tuned case. The next section introduces the experimental setup, consisting of a rectangular cavity where the thermoacoustic instability is mimicked by an electroacoustic feedback loop, to which a Helmholtz resonator can be added. The growth rate of the electro-acoustic instability as well as the purge flow velocity through the damper can be controlled. The results of the analytical predictions and the experiments, as well as results of Simulink simulations of the coupled equations are compared in the last section.

\section{Analytical Model}

\subsection{Derivation of the coupled equations}

Previous work has been done for the derivation of a linear model for the coupling between a damper and an enclosure, see for example $[? 25,26,27,9]$. Throughout the whole paper, $(\cdot)_{d}$ will refer to damper quantities, while $(\cdot)_{n}$ will refer to quantities defined in the damper neck. The standing waves in a cavity (e.g. a combustion chamber) can be expressed with an orthonormal basis $\psi$ of its different acoustic eigenmodes. The pressure at each location within the cavity can be expressed by:

$$
p(t, \mathbf{x})=\sum_{i=1}^{\infty} \eta_{i}(t) \psi_{i}(\mathbf{x})
$$

This is a pre-print version. Published in Journal of Engineering for Gas Turbines and Power (2019) Vol. 141(5), p. 051012 , DOI: $10.1115 / 1.4042080$ 
With $\psi_{i}(\mathbf{x})$ the mode shape and $\eta_{i}(t)$ the amplitude of mode $i$. Assuming that the pressure field is dominated by a certain mode with the highest growth rate and angular frequency $\omega_{0}$, one can neglect the contribution of the other modes, drop the indices and approximate the modal amplitude in frequency domain by [9]:

$$
\begin{aligned}
\hat{\eta}(s) & =\frac{s \rho c^{2}}{s^{2}+\omega_{0}^{2}} \frac{1}{V \Lambda}\left(\frac{\gamma-1}{\rho c^{2}} \int_{V} \hat{Q}(s) \psi^{*}(\mathbf{x}) d V\right. \\
& \left.-\int_{\Sigma-\Sigma_{d}} \hat{\eta}(s) \frac{|\psi(\mathbf{x})|^{2}}{Z(s)} d \Sigma-\int_{\Sigma_{d}} \hat{\eta}(s) \frac{\left|\psi\left(\mathbf{x}_{\mathbf{d}}\right)\right|^{2}}{Z_{d}(s)} d S\right),
\end{aligned}
$$

with $s$ the Laplace variable, $\rho$ the air density, $c$ the speed of sound in the cavity, $V$ the cavity volume, $\Sigma_{d}$ the wall surface equipped with dampers, $Z_{d}$ the damper impedance, $\hat{Q}$ the volumetric source term and $\Lambda$ the spatial norm of the mode defined as:

$$
\Lambda=\frac{1}{V} \int_{V}|\psi|^{2} d V
$$

The second integral in Eq.2 corresponds to the energy flux through the cavity walls and can be modelled by a damping term $\alpha s \hat{\eta}$, with $\alpha>0$ the natural linear damping of the cavity. The first integral, corresponding to the source term, can be similarly modelled by $G e^{-i \omega_{0} \tau} s \hat{\eta}$, with $G$ the gain and $\tau$ the delay between source term and pressure. The linear contribution of the feedback loop to the growth/decay corresponds to the real part of this term $\beta=G \cos \left(\omega_{0} \tau\right)$, which can be either positive or negative. One can then define the growth rate $\nu$ such that $2 \nu=\beta-\alpha$. In the present study, a single damper is used. Its geometry is described in Fig. 1. Using the assumption that the neck diameter is compact with respect to the wavelength, one can assume all quantities to be constant in the third integral. Those simplifications yield:

$$
\hat{\eta}(s)=-\frac{s \rho c^{2}}{s^{2}-2 \nu s+\omega_{0}^{2}} \frac{\Sigma_{d} \hat{\eta}(s)\left|\psi\left(\mathbf{x}_{\mathbf{d}}\right)\right|^{2}}{V \Lambda Z_{d}} .
$$

Multiplying numerator and denominator by the damper back volume $V_{h}=A L$ and the neck length $l$ (end corrections included), using $\Sigma_{d}=a$ to make the damper resonance frequency $\omega_{d}=c \sqrt{a / V_{h} l}$ appear, substituting the damper impedance $Z_{d}$ by $\hat{p} / \hat{u}_{d}=\hat{\eta} \psi\left(\mathbf{x}_{\mathbf{d}}\right) / \hat{u}_{d}$ (with $\hat{u}_{d}$ the acoustic velocity pointing inside the damper), rearranging the terms and switching back to time domain yields our first coupled equation:

$$
\ddot{\eta}-2 \nu \dot{\eta}+\omega_{0}^{2} \eta=-\frac{\varepsilon \omega_{d}^{2} \rho l}{\Psi} \dot{u}_{d}
$$

For ease of notation one defines $\Psi=\psi\left(\mathbf{x}_{\mathbf{d}}\right)$ which describes the maximum mode amplitude at damper location and $\varepsilon$ a damping efficiency factor, depending on the volume ratio and the damper location defined as:

$$
\varepsilon=\frac{V_{h}}{V} \frac{\Psi^{2}}{\Lambda}
$$

With such a model, the pressure amplitude would theoretically grow indefinitely. In practice, however, nonlinear effects stabilize the pressure amplitude to a certain value describing a limit cycle. The minimum order model which can be used to describe such a saturation is the Van der Pol oscillator which is used in our study. This model can represent any nonlinearity that is reasonably approximated by a fourth order Taylor expansion, since the even order terms vanish

This is a pre-print version. Published in Journal of Engineering for Gas Turbines and Power (2019) Vol. 141(5), p. 051012 , DOI: $10.1115 / 1.4042080$ 
when the averaging is performed. The nonlinearities are then simply modelled by a cubic saturation [28], giving:

$$
\begin{aligned}
& \ddot{\eta}-\left(2 \nu+\kappa \eta^{2}\right) \dot{\eta}+\omega_{0}^{2} \eta=-\frac{\varepsilon \omega_{d}^{2} \rho l}{\Psi} \dot{u}_{d} .
\end{aligned}
$$

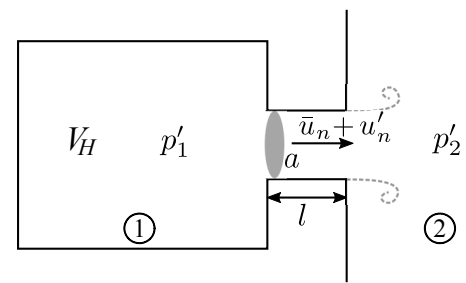

Figure 1: Sketch of a Helmholtz resonator with dimensions. For simplicity the neck length is simply labeled " $l$ " but the model includes a end correction on both sides of the neck

The second coupled equation of the system is now derived using the sketch in Fig. 1. One starts with the momentum equation along a streamline through the damper neck separating between mean and fluctuating quantities for velocity $u=\bar{u}+u^{\prime}$ and pressure $p=\bar{p}+p^{\prime}$ :

$$
\rho \frac{\partial u^{\prime}}{\partial t}+\frac{1}{2} \rho \frac{\partial\left(\bar{u}+u^{\prime}\right)^{2}}{\partial x}+\frac{\partial\left(\bar{p}+p^{\prime}\right)}{\partial x}=0
$$

One integrates in space from (1) to (2), using the neck length compactness (which means $u_{n}^{\prime}$ is constant throughout the neck):

$$
\frac{1}{2} \rho\left(\bar{u}_{1}+u_{1}^{\prime}\right)^{2}+\bar{p}_{1}+p_{1}^{\prime}=\rho l \frac{d u_{n}^{\prime}}{d t}+\frac{1}{2} \rho\left(\bar{u}_{2}+u_{2}^{\prime}\right)^{2}+\bar{p}_{2}+p_{2}^{\prime}
$$

with $l$ the neck length with end corrections. Using the fact that mean quantities verify the Bernoulli equation and deriving with respect to time yields:

$$
\rho\left(\bar{u}_{1}+u_{1}^{\prime}\right) \dot{u}_{1}^{\prime}+\dot{p}_{1}^{\prime}=\rho l \ddot{u}_{n}^{\prime}+\rho\left(\bar{u}_{2}+u_{2}^{\prime}\right) \dot{u}_{2}^{\prime}+\dot{p}_{2}^{\prime}
$$

Since some part of the acoustic energy is dissipated in vortex shedding when a jet occurs as in Fig. 1, the difference between the first order derivative velocity terms on both sides is modelled by introducing a pressure loss coefficient $\zeta$ : if $\bar{u}_{n}+u_{n}^{\prime}>0$ the jet occurs on the right side which is where pressure loss occurs:

$$
\dot{p}_{1}^{\prime}=\rho l \ddot{u}_{n}^{\prime}+\rho \zeta\left(\bar{u}_{n}+u_{n}^{\prime}\right) \dot{u}_{n}^{\prime}+\dot{p}_{2}^{\prime}
$$

Whereas if $\bar{u}_{n}+u_{n}^{\prime}<0$ the jet occurs on the left side:

$$
\rho \zeta\left(\bar{u}_{n}+u_{n}^{\prime}\right) \dot{u}_{n}^{\prime}+\dot{p}_{1}^{\prime}=\rho l \ddot{u}_{n}^{\prime}+\dot{p}_{2}^{\prime}
$$

In addition, using the mass conservation and the equation of state in the volume gives $\dot{p}_{1}^{\prime}=$ $-\rho c^{2} a / V_{h} u_{n}^{\prime}$ and using the fact that $\dot{p}_{2}^{\prime}=\Psi \dot{\eta}$ gives for the whole cycle:

$$
\ddot{u}_{n}^{\prime}+\frac{\zeta}{l}\left|\bar{u}_{n}+u_{n}^{\prime}\right| \dot{u}_{n}^{\prime}+\omega_{d}^{2} u_{n}^{\prime}=-\frac{\Psi}{\rho l} \dot{\eta}
$$

This is a pre-print version. Published in Journal of Engineering for Gas Turbines and Power (2019) Vol. 141(5), p. 051012 , DOI: $10.1115 / 1.4042080$ 


\subsection{Averaging and amplitude equations}

For ease of notation one defines the acoustic velocity in the neck as $u=u_{d}=-u_{n}^{\prime}$. The system of coupled equations is therefore:

$$
\begin{cases}\ddot{\eta}-\left(2 \nu+\kappa \eta^{2}\right) \dot{\eta}+\omega_{0}^{2} \eta=-\frac{\varepsilon \omega_{d}^{2} \rho l}{\Psi} \dot{u} & \kappa<0 \\ \ddot{u}+\frac{\zeta}{l}|\bar{u}+u| \dot{u}+\omega_{d}^{2} u=\frac{\Psi}{\rho l} \dot{\eta} & \zeta>0,\end{cases}
$$

This is a system of two nonlinear oscillators with linear resistive coupling. The $1^{\text {st }}$ oscillator is a Van der Pol oscillator corresponding to self-sustained acoustic oscillations. The $2^{\text {nd }}$ oscillator is a stable nonlinearly damped harmonic oscillator, excited by the derivative of the pressure in front of the damper divided by the mass of air in the damper neck per unit area. As a summary, the main assumptions that were done to derive the equations are the following:

1. Single dominant mode

2. A cubic saturation accurately represents the nonlinearities

3. Neck diameter and length are compact with respect to the mode wavelength

4. Dissipation in the damper is only due to vortex shedding and can thus be represented with a pressure loss coefficient $\zeta$

Following the averaging technique in [29, 30], oscillations are assumed at a frequency $\omega$ unknown but with $\omega \simeq \omega_{0} \simeq \omega_{d}$ and $\omega_{0}+\omega_{d} \simeq 2 \omega$. The ansatz is:

$$
\begin{cases}\eta=A \cos \left(\omega t+\varphi_{A}\right)=\frac{1}{2}\left(a e^{i \omega t}+a^{*} e^{-i \omega t}\right) & a=A e^{i \varphi_{A}} \\ u=B \cos \left(\omega t+\varphi_{B}\right)=\frac{1}{2}\left(b e^{i \omega t}+b^{*} e^{-i \omega t}\right) & b=B e^{i \varphi_{B}}\end{cases}
$$

The first order derivative is:

$$
\begin{aligned}
& \dot{\eta}=\frac{i \omega}{2}\left(a e^{i \omega t}-a^{*} e^{-i \omega t}\right) \quad \text { assuming } \quad \dot{a} e^{i \omega t}+\dot{a}^{*} e^{-i \omega t}=0 \\
& \text { Then } \ddot{\eta}=i \omega \dot{a} e^{i \omega t}-\frac{\omega^{2}}{2}\left(a e^{i \omega t}+a^{*} e^{-i \omega t}\right)
\end{aligned}
$$

A similar sequence is used for $u$ and $b$. Substituting into the first line of Eq. (14), multiplying by $e^{-i \omega t} / i \omega$, simplifying, integrating over one cycle, substituting $a, b$ and $\dot{a}$, dividing by $e^{i \varphi_{A}}$ and taking the real and imaginary part of the equation yields:

$$
\left\{\begin{array}{l}
\dot{A}=\nu A+\frac{\kappa A^{3}}{8}-\frac{\varepsilon \omega_{d}^{2} \rho l}{2 \Psi} B \cos \left(\varphi_{A}-\varphi_{B}\right) \\
\dot{\varphi_{A}}=\frac{\omega_{0}^{2}-\omega^{2}}{2 \omega}+\frac{\varepsilon \omega_{d}^{2} \rho l}{2 \Psi} \frac{B}{A} \sin \left(\varphi_{A}-\varphi_{B}\right)
\end{array}\right.
$$

Similar treatment for the second line of Eq. (14) yields:

$$
\left\{\begin{array}{l}
\dot{B}=\frac{\zeta}{2 l} B^{2} g\left(\frac{\bar{u}}{B}\right)+\frac{\Psi}{2 \rho l} \cos \left(\varphi_{A}-\varphi_{B}\right) \\
\dot{\varphi_{B}}=\frac{\omega_{d}^{2}-\omega^{2}}{2 \omega}+\frac{\Psi}{2 \rho l} \frac{A}{B} \sin \left(\varphi_{A}-\varphi_{B}\right),
\end{array}\right.
$$

This is a pre-print version. Published in Journal of Engineering for Gas Turbines and Power (2019) Vol. 141(5), p. 051012 , DOI: $10.1115 / 1.4042080$ 

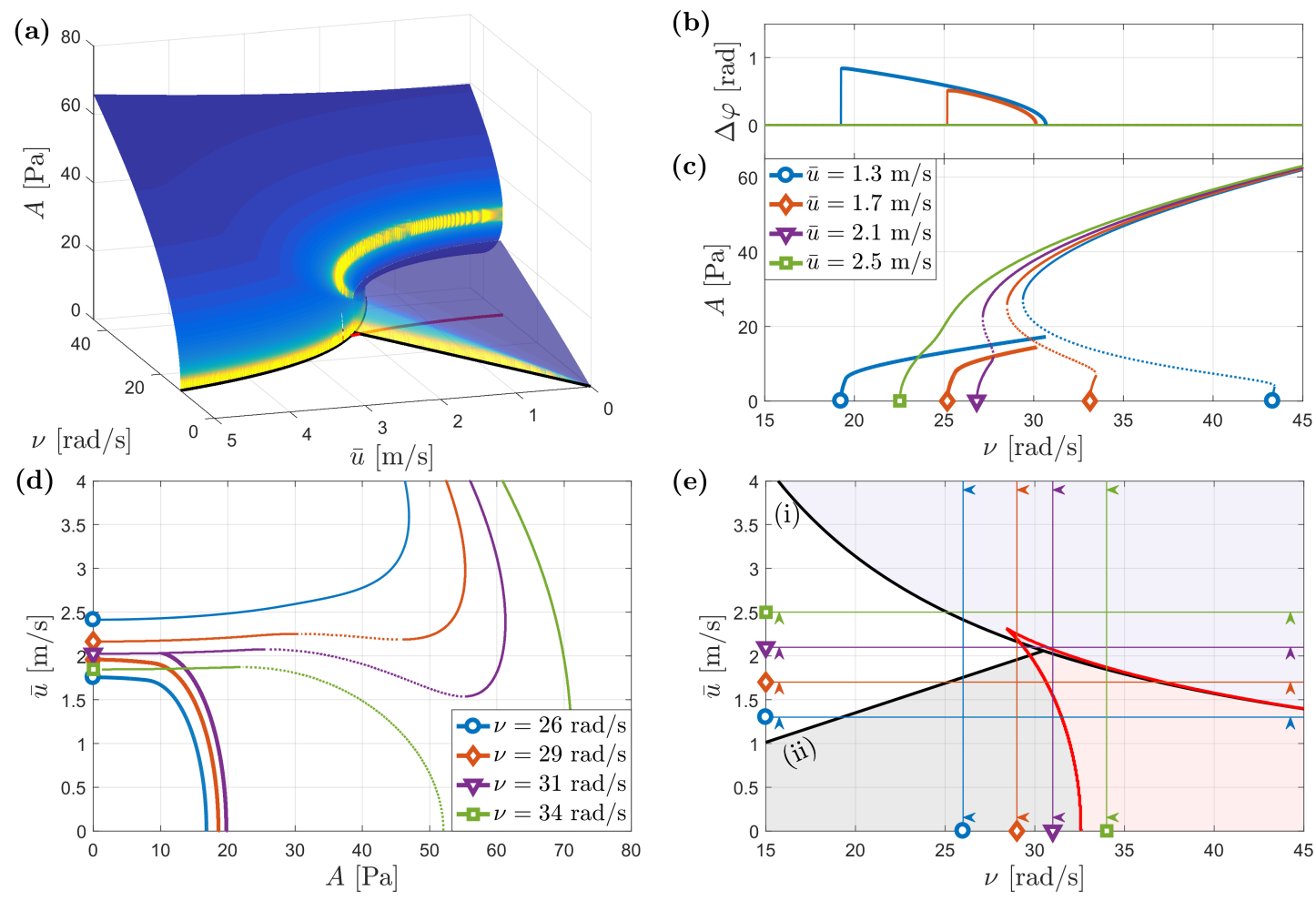

Figure 2: (a) Cavity limit cycle amplitude $A$ as function of $\nu$ and $\bar{u}$ for $\kappa=-0.06\left[\mathrm{~s}^{-1}\right.$.Pa ${ }^{-2}$ (other parameters are the same as in the experimental setup introduced in the next section) Coloring: gradient of the amplitude $A$. The transparent surface corresponds to the solutions of Eq. (25) ( $\eta$ and $u$ not in phase), while the opaque surface corresponds to the solutions of Eq. (22) ( $\eta$ and $u$ in phase) (b,c) Corresponding bifurcation diagram as function of $\nu$ for different fixed $\bar{u}$ for the amplitude $A$ (c) and the phase $\Delta \varphi$ (b). Plain lines correspond to stable fixed points (i.e. limit cycles. thick=out-of-phase, thin=in phase), dotted lines correspond to unstable fixed points. (d) Corresponding bifurcation diagram as function of $\bar{u}$ for different fixed $\nu$. (e) Stability map as function of $\nu$ and $\bar{u}$. Black lines: Hopf bifurcations corresponding to the linear model stability limit. Red lines: fold bifurcations merging in a cusp catastrophe point at $(\nu=28.4 \mathrm{rad} / \mathrm{s}, \bar{u}=2.31 \mathrm{~m} / \mathrm{s})$. Grey shaded area: limit-cycles corresponding to out-of-phase solution. Pink shaded area: zone of existence of an unstable fixed point. Purple shaded area: zone where a single stable limit cycle exists. The area not shaded corresponds to the zone where the whole system is stable.

This is a pre-print version. Published in Journal of Engineering for Gas Turbines and Power (2019) Vol. 141(5), p. 051012, DOI: $10.1115 / 1.4042080$ 
with the following function coming from the averaging of the damping term with absolute value:

$$
g(x)=\left\{\begin{array}{lll}
\frac{2}{\pi}\left(x \arcsin (x)+\left(2+x^{2}\right) \frac{\sqrt{1-x^{2}}}{3}\right) & \text { for } & |x| \leq 1 \\
x & \text { for } & |x|>1
\end{array}\right.
$$

This function corresponds to the one in the nonlinear damping term model used in $[15,16 ?$ ], whose accuracy will be confirmed experimentally in the next section. Defining $\Delta \varphi=\varphi_{A}-\varphi_{B}$ yields the following system of three coupled equations for the slowly varying amplitudes and the phase between the coupled oscillators:

$$
\left\{\begin{array}{l}
\dot{A}=\nu A+\frac{\kappa A^{3}}{8}-\frac{\varepsilon \omega_{d}^{2} \rho l}{2 \Psi} B \cos (\Delta \varphi) \\
\dot{B}=-\frac{\zeta}{2 l} B^{2} g\left(\frac{\bar{u}}{B}\right)+\frac{\Psi}{2 \rho l} A \cos (\Delta \varphi) \\
\Delta \dot{\varphi}=\Delta \omega+\left(\frac{\varepsilon \omega_{d}^{2} \rho l}{2 \Psi} \frac{B}{A}-\frac{\Psi}{2 \rho l} \frac{A}{B}\right) \sin (\Delta \varphi)
\end{array}\right.
$$

with $\Delta \omega=\frac{\omega_{0}^{2}-\omega_{d}^{2}}{2 \omega} \simeq \omega_{0}-\omega_{d}$ the detuning constant.

\subsection{Solutions and interpretation}

For the solutions to be partially analytically tractable, one has to assume that the damper is perfectly tuned, i.e. $\Delta \omega=0$. The first trivial fixed point of the system in Eq. (20) is $(0,0,0)$. For the non-trivial fixed points, there are two types of solutions. Either (i) pressure and velocity are in phase, i.e. $\Delta \varphi=0$. Then

$$
A=\frac{\zeta \rho}{\Psi} B^{2} g\left(\frac{\bar{u}}{B}\right)
$$

Plugging this into the second line of Eq. (20) gives:

$$
B^{5} g^{3}\left(\frac{\bar{u}}{B}\right)+\frac{8 \nu \Psi^{2}}{\kappa \zeta^{2} \rho^{2}} B g\left(\frac{\bar{u}}{B}\right)-\frac{4 \varepsilon l \Psi^{2} \omega_{0}^{2}}{\kappa \zeta^{3} \rho^{2}}=0
$$

Depending on the values of the parameters $\kappa, \nu$ and $\bar{u}$, this equation yields between 0 and 3 solutions. The other type of solution can be found if (ii) pressure and velocity are not in phase, i.e. $\Delta \varphi \neq 0$ :

$$
\begin{aligned}
& \frac{\varepsilon \omega_{d}^{2} \rho l}{2 \Psi} \frac{B}{A}-\frac{\Psi}{2 \rho l} \frac{A}{B}=0 \\
& \Rightarrow A=\frac{\omega_{d} \rho l \sqrt{\varepsilon}}{\Psi} B
\end{aligned}
$$

Plugging this into the first line of Eq. (20) gives:

$$
\cos (\Delta \varphi)=\frac{\kappa}{4} \omega_{d} \rho^{2} l^{2} \sqrt{\varepsilon} B^{2}+\frac{2 \nu}{\omega_{d} \sqrt{\varepsilon}}
$$

This is a pre-print version. Published in Journal of Engineering for Gas Turbines and Power (2019) Vol. 141(5), p. 051012 , DOI: $10.1115 / 1.4042080$ 
Plugging the previous results into the second line of Eq. (20) gives:

$$
-\frac{\zeta}{2 l} B g\left(\frac{\bar{u}}{B}\right)+\frac{\kappa}{8} \frac{\omega_{d}^{2} \rho^{2} l^{2} \varepsilon}{\Psi^{2}} B^{2}+\nu=0
$$

Depending on the values of the parameters $\kappa, \nu$ and $\bar{u}$, this equation yields either 0 or 1 solution. Equations (22) and (25) are numerically solved for velocity amplitude $B$ and the results used with the other equations to obtain $A$ and $\Delta \varphi$. Since only the acoustic pressure will be measured in the real cavity case, the limit cycles are described in terms of averaged amplitude $A$ only. The solutions correspond to stable or unstable fixed points of the system. The stability of the fixed points has been determined by looking at the signs of the eigenvalues of the Jacobian of Eq. 20 at the values of the fixed points. In Figure 2(c) and (d), the stable fixed points (i.e. limit cycles) are represented by plain lines, and the unstable fixed points by dotted lines. The fixed points corresponding to solutions of Eq.25 (out-of-phase) are always stable and are represented by thicker plain lines.

Figure 2 presents the analytical results for pressure amplitude $A$ for one fixed $\kappa=-0.06$ $\left[\mathrm{s}^{-1} \cdot \mathrm{Pa}^{-2}\right]$. First, a $3 \mathrm{D}$ view of the cavity limit cycle amplitude $A$ as function of $\nu$ and $\bar{u}$ is shown in Fig. 2 (a). The transparent surface corresponds to the solutions of Eq. (25) ( $\eta$ and $u$ not in phase), while the opaque surface corresponds to the solutions of Eq. (22) ( $\eta$ and $u$ in phase). Taking a closer look at the bifurcations in 2D (Fig. 2 (e)), the two Hopf bifurcations (black lines, defined as transition from stable system to a limit cycle) correspond to the stability limits predicted by the linear model: indeed if one starts from Eq. 4, considers the system excited by non-coherent noise, and uses a linear impedance model for the damper, then the RHS corresponds to a linear transfer function. Applying the Routh-Hurwitz criterion to this transfer function gives the linear stability limits corresponding to the Hopf bifurcations in Fig. 2 (e) (see e.g. [? ]). The result of the use of a nonlinear damping model is the appearance of the two fold bifurcations curves (in red), which are defined as loci where two fixed points collide and disappear. For the present case, they are equivalent to the limit of existence of an unstable fixed point. The point where those two fold bifurcation curves meet is called a cusp catastrophe point, occuring at $(\nu=28.4 \mathrm{rad} / \mathrm{s}, \bar{u}=2.31$ $\mathrm{m} / \mathrm{s})$. The non-shaded area then corresponds to the stable zone. The grey-shaded area corresponds to a single out-of-phase limit-cycle. The pink-shaded area is the zone of existence of an unstable fixed point, and the purple-shaded area is where a single stable in-phase limit cycle exists.

Some cuts of the bifurcation diagram in Fig. 2 (e) are shown in Fig. 2 (d) for fixed $\nu$ and Fig. 2 (b) and (c) for $A$ and $\Delta \varphi$ for fixed $\bar{u}$ respectively. Taking a closer look at Fig. 2 (c) since those are the results which will be compared to simulations and experiments: one can see that there is an "ideal" purge flow velocity $\bar{u} \simeq 2.1 \mathrm{~m} / \mathrm{s}$ for which the onset of instability occurs at the highest initial growth rate $\nu$ possible (here about $30 \mathrm{rad} / \mathrm{s}$ ). For fixed velocity $\bar{u}<2.1 \mathrm{~m} / \mathrm{s}$, and an increasing $\nu$, a first limit cycle develops when crossing the Hopf bifurcation line (ii) for which $\eta$ and $u$ are not in phase, with the phase difference decreasing progressively as one increases $\nu$ (see Fig. 2 (b)), until it reaches the fold bifurcation, at which the system "jumps" to a higher limit cycle for which $\eta$ and $u$ are in phase. From the shape of the bifurcation plots in Fig. 2 (c), one might also expect some hysteresis to happen when the growth rate $\nu$ is ramped up and down.

\section{Experiments}

\subsection{Validation of the damper nonlinear model}

First the validity of the nonlinear damper model using the function $g$ in Eq. (19) has to be confirmed. In order to achieve this, the acoustic reflection coefficient $\mathcal{R}$ of our Helmholtz resonator

This is a pre-print version. Published in Journal of Engineering for Gas Turbines and Power (2019) Vol. 141(5), p. 051012 , DOI: $10.1115 / 1.4042080$ 

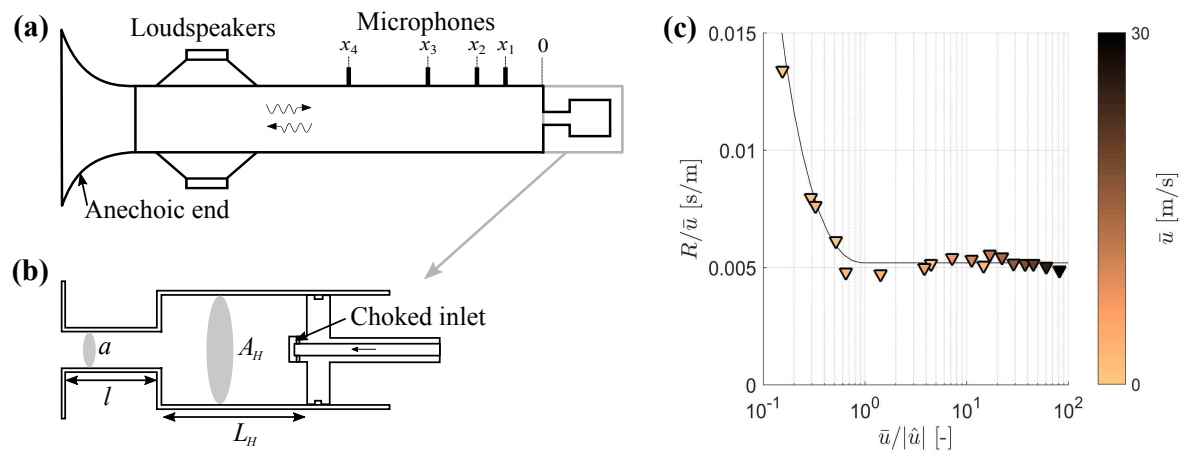

Figure 3: (a) Sketch of the impedance tube used for the damper nonlinear model validation (b) cut of the Helmholtz damper (c) Comparison between the resistive term obtained from a fit on the experimental reflection coefficient (triangles) and the resistive term obtained from the nonlinear model $R_{N L}$ (black line).

was measured for different mean purge flow velocities and different excitation amplitudes using the Multi-Microphone-Method[31]. A sketch of the impedance tube can be seen in Fig. 3(a). Two Beyma 12SW1300Nd Loudspeakers and four G.R.A.S. 46BD 1/4" CCP microphones were used. The impedance tube cross-section is $62 \times 62 \mathrm{~mm}$. The damper is also sketched in Fig. 3(b), and its dimensions are as follows: the neck has a diameter of $16.4 \mathrm{~mm}$ and a length of $45 \mathrm{~mm}$. The appropriate end correction is determined using the Helmholtz solver AVSP for the corresponding geometry, which gives $l_{\text {cor }}=15.6 \mathrm{~mm}$. The back-cavity of the Helmholtz damper is $64 \mathrm{~mm}$ long (this length is adjustable to be able to tune the damper) and $50 \mathrm{~mm}$ of diameter. Eight small choked holes feed the damper with purge flow. The experimental reflection coefficient is used to compute the acoustic impedance of the damper using

$$
Z_{d}=\frac{\rho c+\mathcal{R}}{\rho c-\mathcal{R}}
$$

This experimental impedance is fitted to a simple model by adjusting the specific resistance term $R$ (non-frequency dependent):

$$
Z_{d}=\rho c\left(\frac{l}{c} \cdot \frac{s^{2}+\omega_{d}^{2}}{s}+R\right),
$$

Finally this experimentally fitted resistance $R$ is compared to the resistive term from the nonlinear model: indeed, starting from Eq. 13, performing a Fourier series expansion of the nonlinear term while neglecting higher frequency terms, uses $\Psi \dot{\eta}=\dot{p}$, and switching back to frequency domain yields the same expression as Eq. 27 replacing $R$ by $R_{N L}$ defined as follows:

$$
R_{N L}=\frac{\zeta \hat{u}}{c} g\left(\frac{\bar{u}}{\hat{u}}\right)
$$

with $\hat{u}$ the acoustic velocity and $g$ the function from Eq. (19). This model with non-frequency dependent nonlinear resistive term has already been used in $[15,16 ?]$. The value of the pressure loss coefficient $\zeta$ has to be fitted so that $R_{N L}$ best matches the resistive term computed for each single measure. The comparison is done in Fig. 3(c) for $\zeta=1.78$ which gives very good agreement, whether on the linear part (straight line) or on the nonlinear part.

This is a pre-print version. Published in Journal of Engineering for Gas Turbines and Power (2019) Vol. 141(5), p. 051012 , DOI: $10.1115 / 1.4042080$ 


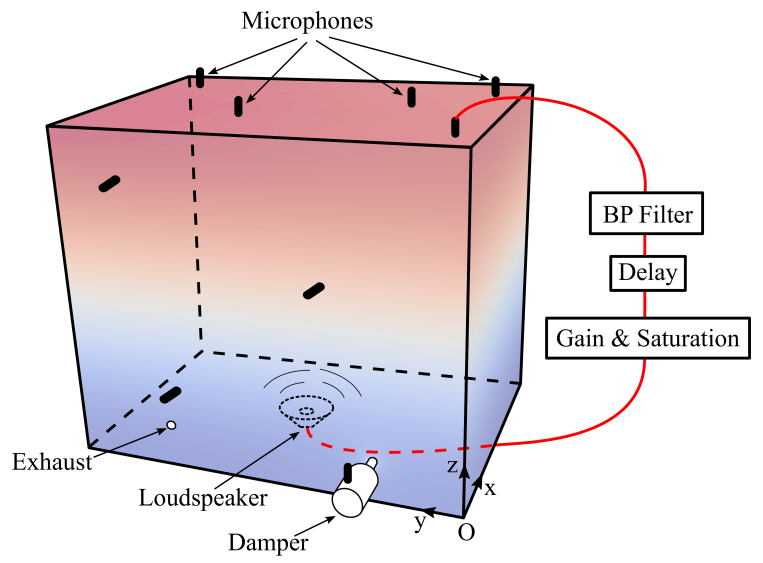

Figure 4: Sketch of the measurement setup

\begin{tabular}{|c|c|c|c|}
\hline Element & $x$ & $y$ & $z$ \\
\hline Feedback Microphone & 70 & 80 & 590 \\
Feedback Loudspeaker & 245 & 345 & 50 \\
Damper & 0 & 165 & 45 \\
Exhaust & 0 & 325 & 45 \\
\hline
\end{tabular}

Table 1: Coordinates of the different elements in $\mathrm{mm}$ in the cavity coordinate system

\subsection{Cavity-damper coupled system measurements}

To show the relevance of the analytical model from the first section, a coupled damper-cavity resonance experiment has been designed. The experimental setup consists of a main cavity, which is a $500 \mathrm{~mm} \times 700 \mathrm{~mm} \times 600 \mathrm{~mm}$ parallelepipoid metal box, with ridges on the side panels and tensioned metal rods between both main panels to guarantee rigidity. This is essential to ensure that the measured resonances are indeed acoustic resonances and that no mechanical resonance interferes. A Pioneer TS-1001I loudspeaker, mounted inside the cavity, is part of the feedback loop that creates the instability. The hole through which the cable enters the cavity also serves as an exhaust for the damper purge flow. A set of eight G.R.A.S. 46BD 1/4" CCP microphones are distributed at different locations on the cavity boundaries for measuring pressure. One suitably located microphone is chosen as the feedback microphone: its signal is filtered, delayed, saturated and amplified by a LabVIEW program loaded onto the National Instruments cRIO-9066 board before being sent back to the loudspeaker. The feedback loop which mimics the constructive interaction between flame heat release and acoustic pressure occurring in real combustion systems is thus completed. The filter is necessary to ensure that only the first transversal eigenmode (mode of interest) is excited. This mode has a frequency of about $288 \mathrm{~Hz}$ and a natural damping $\alpha=-4.8$ $\mathrm{rad} / \mathrm{s}$. The additional acoustic losses induced by the exhaust hole when flow is passing through gives an increase of $0.4 \mathrm{rad} / \mathrm{s}$ to this damping, which is negligible compared to the growth rates $\nu$ at which the onset of instability occurs. One can then see in Fig. 4 that both the loudspeaker and the feedback microphone are located at pressure anti-nodes. The exact location of the different elements in the cavity coordinate system is shown in Table 1 . The delay $\tau=2.1 \mathrm{~ms}$, giving the most unstable state (i.e. maximizing $\beta$ ) was chosen and kept fixed for the whole set of experiments.

This is a pre-print version. Published in Journal of Engineering for Gas Turbines and Power (2019) Vol. 141(5), p. 051012 , DOI: $10.1115 / 1.4042080$ 

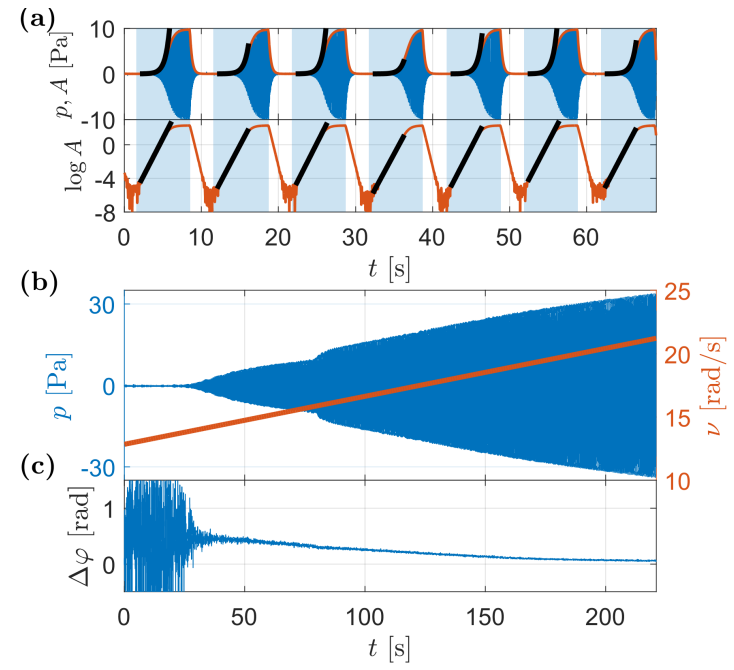

Figure 5: Measurement techniques: growth rate measurements (a) and growth rate ramping with effect on the pressure inside the cavity (b) and on the phase between the cavity pressure and the damper neck velocity (which corresponds to $\pi / 2$ substracted to the phase between cavity pressure and damper volume pressure)

The saturation takes the form of the nonlinear term of the first coupled equation, similar to what was used in [32]:

$$
S(U)=c_{1} U+c_{2} U^{3},
$$

with $U$ the incoming voltage. One can thus play on the saturation coefficient and the growth rate separately, knowing that $\nu_{\text {eff }} \propto c_{1}$ and $\kappa_{\text {eff }} \propto c_{2}$. On one of the cavity main panels, the previously tested Helmholtz damper can be mounted and fed with a purge flow. From the location of the damper with respect to the mode $\Psi \simeq 0.95$ is an adequate estimation. An additional microphone records the pressure inside the damper volume, which is in quadrature with the velocity $u$ inside the damper neck.

Two types of measurements were performed and are shown in Fig. 5. To determine the law between $c_{1}$ and $\nu$, growth rate measurements were performed where one lets the instability grow and then fits the linear growth of the logarithm of the envelope and average over ten instances to obtain the growth rate (Fig. 5 (a)). Once the growth rate is known, one can get the actual saturation coefficient $\kappa$ from any limit cycle amplitude without damper using: $A=\sqrt{-8 \nu / \kappa}$. The second type of measurement performed is the actual ramping of the growth rate, which was achieved by simply ramping $c_{1}$ automatically within the LabVIEW program. The experimental ramp speed was about $20 \mathrm{~s}$ for $1 \mathrm{rad} / \mathrm{s}$ increase/decrease. An example of the results of such a measurement is shown Figs. 5 (b) and (c) for $\bar{u}=1.3 \mathrm{~m} / \mathrm{s}$ : one can see that the pressure inside the cavity grows to a limit cycle past a certain growth rate $\nu$, and then jumps to a higher limit cycle. The phase between cavity pressure and damper neck velocity (obtained by substracting $\pi / 2$ to the phase between damper volume pressure and cavity pressure) also follows the trend shown in Fig. 2 (b): it is not defined when there is no limit cycle, then starts from a non-zero value and decays to zero as the growth rate is increased.

This is a pre-print version. Published in Journal of Engineering for Gas Turbines and Power (2019) Vol. 141(5), p. 051012, DOI: $10.1115 / 1.4042080$ 


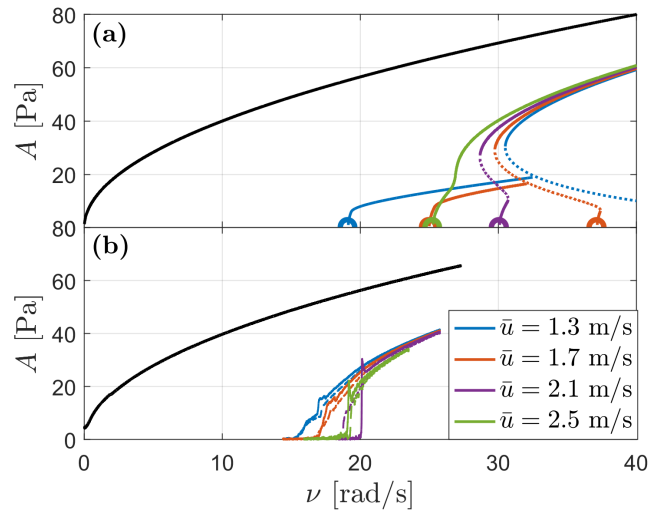

Figure 6: Comparison between analytical results (a) and experiments (envelope of the pressure measured during ramping of $\nu$, plain=ramping up, dashed=ramping down) (b). Black lines= cavity without damper. Besides the mismatch for the onset growth rate, one can see that the tendency is well reproduced, and all curves seem to collapse for higher growth rate even though their order is reversed.

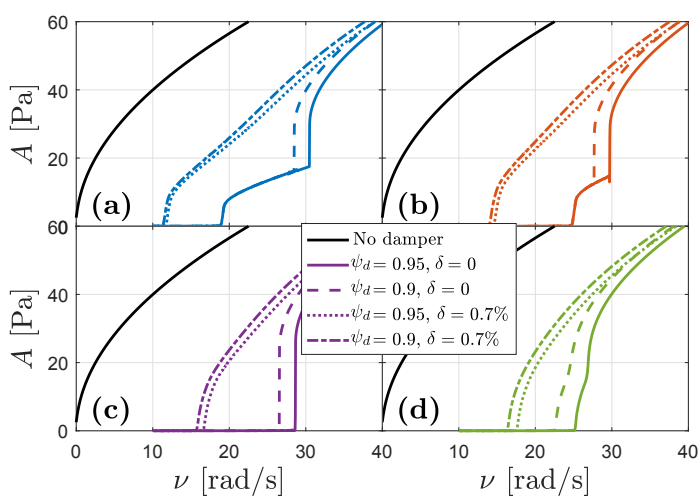

Figure 7: Comparison between numerical results from slowly ramping down the growth rate, highlighting the influence of detuning $\delta$ and of damper position with regard to mode shape $\Psi$ (a) $\bar{u}=1.3 \mathrm{~m} / \mathrm{s}$ (b) $\bar{u}=1.7 \mathrm{~m} / \mathrm{s}$ (c) $\bar{u}=2.1 \mathrm{~m} / \mathrm{s}(\mathrm{d}) \bar{u}=2.5 \mathrm{~m} / \mathrm{s}$

This is a pre-print version. Published in Journal of Engineering for Gas Turbines and Power (2019) Vol. 141(5), p. 051012, DOI: $10.1115 / 1.4042080$ 


\section{Results}

We now concentrate on the effect of the damper on the cavity pressure amplitude $A$ only. Figure 6 compares the results of the analytical model (top) with the experiments (bottom) for different purge flow velocities $\bar{u}$. The results of the cavity without damper (black curves) are very well reproduced. For the results with damper, the behavior of the limit cycles is also well reproduced although there is a $30 \%$ error on the predicted onset of instability between model and experiments. Our hypothesis is that this is due to a small detuning of the damper: indeed, modifying the damper back cavity length $L$ by $1 \mathrm{~mm}$ leads to a detuning $\Delta \omega / \omega_{0}>0.7 \%$. Figure 7 shows how such a small detuning can strongly influence the onset point of the instability. Another source of uncertainty is the maximum mode amplitude at damper location $\Psi$. Since the cavity acoustic shape is altered by the presence of the loudspeaker, the exhaust hole and the damper itself it can well be that the mode shape is different from the theoretical one and that $\Psi$ has been overestimated. Despite this fact, in Fig. 6, the onset of instability follows the trend of the model for increasing velocity: at first, the higher the purge flow, the later the instability is triggered, until one reaches an "ideal" velocity, after which the onset of instability occurs at lower initial growth rates again. The collapsing of all curves for high growth rates is also well reproduced even though their order is reversed.

In addition to the analytical work and to the experiments, Simulink simulations of the system in Eq. (14) were performed for different $\bar{u}$ ramping $\nu$ up and down at a rate of about 100 s for $1 \mathrm{rad} / \mathrm{s}$ increase/decrease. In order to have a better look at the limit cycle behavior close to the onset of instability, one has to zoom on this part of the curve for both Simulink/analytical model and experiments. Those results for ramping up and down and for different velocities are shown in Fig. 8. The agreement between theoretical results and simulations (on the left) is very good. One is able to reproduce the "jump" between limit cycles $((\mathbf{c}),(\mathbf{e}),(\mathbf{g})$ and (i)), and the inferred hysteresis as well $((\mathbf{k})$ and $(\mathbf{m}))$. And most importantly, the general behaviour of the experimental limit cycles (on the right) confirms the validity of our model since both the "jump" from different limit cycles ((d) and (f)), and the hysteresis ((l) and (n)) are reproduced.

\section{Conclusion}

An analytical model for the cavity-damper coupled system was derived, using a linearly unstable oscillator with cubic saturation for the cavity and a nonlinear damping for the damper. Amplitude equations were derived and the theoretical limit cycles of the coupled system computed in the case where the damper is perfectly tuned. Simulink simulations were performed which are in excellent agreement with the theoretical predictions. Experiments were then performed by ramping the growth rate inside a rectangular cavity with a damper, in which the thermoacoustic instability is mimicked by an electro-acoustic feedback loop. Although the onset of instability occured about $30 \%$ earlier than expected (most probably due to slight detuning of the damper), some of the nonlinear features of the model were experimentally reproduced, thus underlining the importance of using a nonlinear model for the damper. This is essential if one wants to be able to predict potential dangerous behavior close to the onset of instability when mapping a new engine for example, such as hysteresis and sudden "jumps" from one limit cycle to another.

\section{References}

[1] J. Keller, Thermoacoustic oscillations in combustion chambers of gas turbines, AIAA Journal 33 (12) (1995) 2280-2287.

This is a pre-print version. Published in Journal of Engineering for Gas Turbines and Power (2019) Vol. 141(5), p. 051012 , DOI: $10.1115 / 1.4042080$ 
Simulink
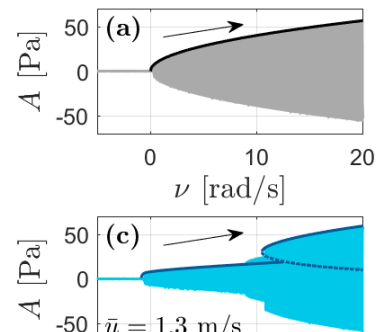

$-50-\bar{u}=1.3 \mathrm{~m} / \mathrm{s}$
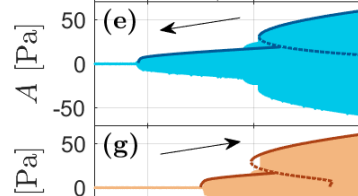

${ }_{-50}-\bar{u}=1.7 \mathrm{~m} / \mathrm{s}$

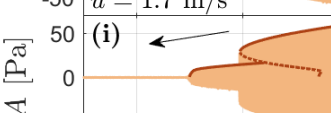

$\checkmark-50$

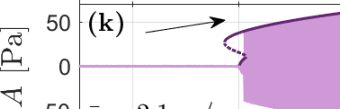

$-50-\bar{u}=2.1 \mathrm{~m} / \mathrm{s}$
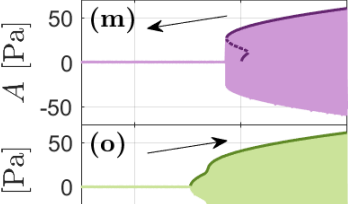

$-50-\bar{u}=2.5 \mathrm{~m} / \mathrm{s}$

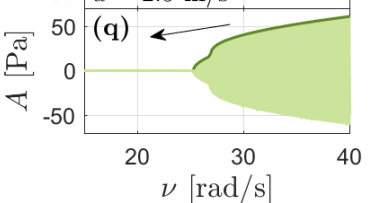

Experiments
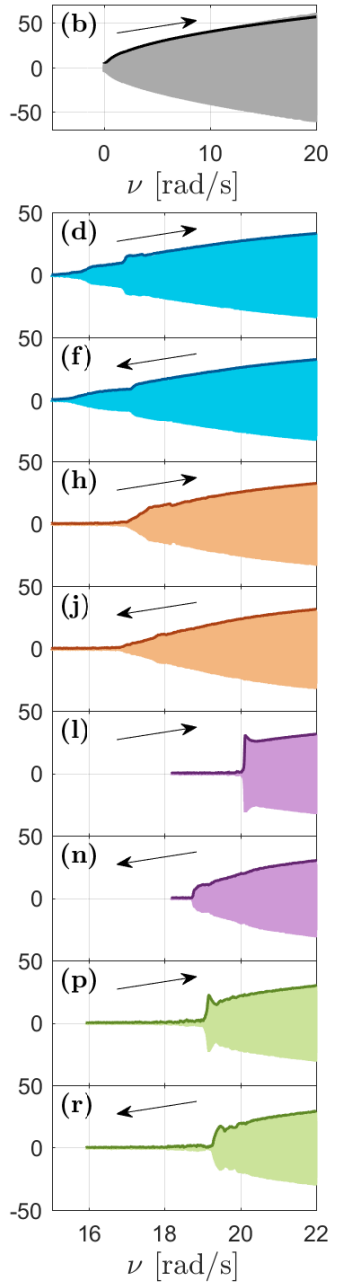

Figure 8: Comparison between Simulink simulations and analytical model (left column) and experiments (right column). On the left, the results of the analytical model are superimposed on the Simulink simulations, which highlights the perfect match between both. (a) and (b) represent the cavity without damper. Despite a different onset of instability, nonlinear features are well reproduced experimentally, for example the "jump" in (d) and (f), and the hysteresis in (1) and (n).

This is a pre-print version. Published in Journal of Engineering for Gas Turbines and Power (2019) Vol. 141(5), p. 051012, DOI: $10.1115 / 1.4042080$ 
[2] T. C. Lieuwen, Experimental investigation of limit-cycle oscillations in an unstable gas turbine combustor, J. Propul. Power 18 (1) (2002) 61-67.

[3] S. Stow, A. Dowling, Low-order modelling of thermoacoustic limit cycles, ASME Paper No. GT2004-54245.

[4] S. Stow, A. Dowling, A time-domain network model for nonlinear thermoacoustic oscillations, in: ASME Turbo Expo 2008: Power for Land, Sea, and Air, American Society of Mechanical Engineers, 2008, pp. 539-551.

[5] D. Zhao, X. Li, A review of acoustic dampers applied to combustion chambers in aerospace industry, Prog. Aerosp. Sci. 74 (2015) 114-130.

[6] I. Dupère, A. Dowling, The use of Helmholtz resonators in a practical combustor, J. Eng. Gas Turbines Power 127 (2) (2005) 268-275.

[7] M. Bothien, N. Noiray, B. Schuermans, A novel damping device for broadband attenuation of low-frequency combustion pulsations in gas turbines, J. Eng. Gas Turbines Power 136 (4) (2014) 041504.

[8] G. Mensah, J. Moeck, Acoustic damper placement and tuning for annular combustors: An adjoint-based optimization study, J. Eng. Gas Turbines Power 139 (6) (2017) 061501.

[9] N. Noiray, B. Schuermans, Theoretical and experimental investigations on damper performance for suppression of thermoacoustic oscillations, J. Sound Vib. 331 (12) (2012) 27532763.

[10] K. Förner, A. Cárdenas-Miranda, W. Polifke, Mapping the influence of acoustic resonators on rocket engine combustion stability, J. Propul. Power.

[11] M. Zahn, M. Betz, M. Wagner, N. Stadlmair, M. Schulze, C. Hirsch, T. Sattelmayer, Impact of Damper Parameters on the Stability Margin of an Annular Combustor Test Rig, in: Proc. ASME Turbo Expo 2017, 2017, p. V04BT04A001.

[12] G. Campa, S. Camporeale, Influence of nonlinear effects on the limit cycle in a combustion chamber equipped with Helmholtz resonator, in: ASME Turbo Expo, 2014.

[13] C. Tam, K. Kurbatskii, K. Ahuja, R. Gaeta, A numerical and experimental investigation of the dissipation mechanisms of resonant acoustic liners, J. Sound Vib. 245 (3) (2001) 545-557.

[14] A. Hersh, B. Walker, J. Celano, Helmholtz resonator impedance model, part 1: Nonlinear behavior, AIAA Journal 41 (5) (2003) 795-808.

[15] B. Zinn, A theoretical study of non-linear damping by Helmholtz resonators, J. Sound Vib. 13 (3) (1970) 347-356.

[16] J. Keller, E. Zauner, On the use of Helmholtz resonators as sound attenuators, ZAMP Zeitschrift für Angew. Math. und Phys. 46 (3) (1995) 297-327.

[17] D. Singh, S. Rienstra, Nonlinear asymptotic impedance model for a Helmholtz resonator liner, J. Sound Vib. 333 (15) (2014) 3536-3549.

[18] K. Förner, J. Tournadre, W. Polifke, P. Martínez-Lera, Characterization of the nonlinear response of a Helmholtz resonator, SFB/TRR40 Annu. Rep. 2015.

[19] J. Tournadre, K. Förner, P. Martínez-Lera, W. Polifke, W. Desmet, Determination of Acoustic Impedance for Helmholtz Resonators Through Incompressible Unsteady Flow Simulations, 22nd AIAA/CEAS Aeroacoustics Conf.

[20] I. Park, C. Sohn, Nonlinear acoustic damping induced by a half-wave resonator in an acoustic chamber, Aerosp. Sci. Technol. 14 (6) (2010) 442-450.

[21] B. Ćosić, T. Reichel, C. Paschereit, Acoustic response of a Helmholtz resonator exposed to hot-gas penetration and high amplitude oscillations, J. Eng. Gas Turbines Power 134 (10) (2012) 101503.

[22] B. Ćosić, D. Wassmer, S. Terhaar, C. Paschereit, Acoustic response of Helmholtz dampers in

This is a pre-print version. Published in Journal of Engineering for Gas Turbines and Power (2019) Vol. 141(5), p. 051012 , DOI: $10.1115 / 1.4042080$ 
the presence of hot grazing flow, J. Sound Vib. 335 (2015) 1-18.

[23] M. Bothien, D. Wassmer, Impact of density discontinuities on the resonance frequency of helmholtz resonators, AIAA Journal 53 (4) (2015) 877-887.

[24] D. Yang, A. Morgans, Acoustic models for cooled helmholtz resonators, AIAA Journal 55 (9) (2017) 3120-3127.

[25] F. Fahy, C. Schofield, A note on the interaction between a Helmholtz resonator and an acoustic mode of an enclosure, J. Sound Vib. 72 (3) (1980) 365-378.

[26] A. Cummings, Effects of a resonator array on the sound field in a cavity, J. Sound Vib. 154 (1) (1992) 25-44.

[27] D. Li, L. Cheng, Acoustically coupled model of an enclosure and a Helmholtz resonator array, J. Sound Vib. 305 (1-2) (2007) 272-288.

[28] N. Noiray, A. Denisov, A method to identify thermoacoustic growth rates in combustion chambers from dynamic pressure time series, Proc. Combust. Inst. 36 (3) (2017) 3843-3850.

[29] R. Stratonovich, Topics in the theory of random noise, Vol. 2, CRC Press, 1967.

[30] A. Balanov, N. Janson, D. Postnov, O. Sosnovtseva, Synchronization: from simple to complex, Springer, 2009.

[31] S.-H. Jang, J.-G. Ih, On the multiple microphone method for measuring in-duct acoustic properties in the presence of mean flow, J. Acoust. Soc. Am. 103 (3) (1998) 1520-1526.

[32] N. Noiray, B. Schuermans, Deterministic quantities characterizing noise driven Hopf bifurcations in gas turbine combustors, Int. J. Non Linear Mech. 50 (2013) 152-163.

This is a pre-print version. Published in Journal of Engineering for Gas Turbines and Power (2019) Vol. 141(5), p. 051012 , DOI: $10.1115 / 1.4042080$ 